\title{
Personalized medicine in a dish: the growing possibility of neuropsychiatric disease drug discovery tailored to patient genetic variants using stem cells
}

\author{
Kristen J. Brennand \\ Departments of Genetics and Genomics, Neuroscience, and Psychiatry, Icahn School of Medicine at Mount Sinai, New York, NY 10029, USA \\ Correspondence to: Kristen J. Brennand. Departments of Genetics and Genomics, Neuroscience, and Psychiatry, Icahn School of Medicine at Mount \\ Sinai, New York, NY 10029, USA. Email: kristen.brennand@mssm.edu. \\ Provenance: This is an invited Editorial commissioned by Editor-in-Chief Zhizhuang Joe Zhao (Pathology Graduate Program, University of \\ Oklahoma Health Sciences Center, Oklahoma City, USA). \\ Comment on: Lorenz C, Lesimple P, Bukowiecki R, et al. Human iPSC-derived neural progenitors are an effective drug discovery model for \\ neurological mtDNA disorders. Cell Stem Cell 2017;20:659-74.e9.
}

Received: 06 October 2017; Accepted: 27 October 2017; Published: 16 November 2017.

doi: $10.21037 /$ sci.2017.10.05

View this article at: http://dx.doi.org/10.21037/sci.2017.10.05

The mechanistic links between patient genotype and the phenotypic changes associated with neuropsychiatric disease have been difficult to establish, owing in part to the lack of live brain tissue from clinical cases. In fact, for many brain disorders, it remains unclear whether disease progression reflects developmental aberrations during neural differentiation or activity-dependent perturbations in mature neurons. Fortunately, the ability to reprogram cells from patients and healthy controls into human induced pluripotent stem cells (hiPSCs) (1) has conferred the ability to generate a nearly limitless source of genetically matched human neural cells with which to model neuropsychiatric disease across a variety of neural cell fates. Already, hiPSCbased models have provided molecular and cellular insights into disease mechanisms underlying far-ranging brain disorders from autism spectrum disorder (2) to schizophrenia (3), Alzheimer's disease (4), Parkinson's disease (5), and even zika-virus induced microcephaly (6). These models are being increasingly applied to drug screening, successfully identifying compounds to enhance neural proliferation (7), modulators of lithium signaling (8) and inhibitors of zika virus infection (9). While many of these early screens have been conducted on neural progenitor cells (NPCs), screening has recently been extended to stem cell-derived neurons and astrocytes (10-12).

Because work by ourselves and others demonstrate that current hiPSC differentiation strategies yield neurons that most resemble fetal brain cells (13-16), hiPSC-based models remain best suited for the study of disease predisposition. Consistent with this, hiPSC-based studies of late onset neurodegenerative diseases such as Parkinson's disease (5), Alzheimer's disease (4) and amyotrophic lateral sclerosis (17) have failed to recapitulate the severe neuronal loss observed in human disease. Simply put, neural cells generated from patient-specific hiPSCs capture the genetic risk factors, known and unknown, that a given individual was born with, but fall short of modeling the complex cellular interactions and circuit-based activity that contribute to disease initiation or progression. As a result, we and others have developed and validated models of autism spectrum disorder (18), schizophrenia $(13,19,20)$, bipolar disorder (21), Parkinson's disease (22) and Huntington's disease (23) that focus on the molecular and cellular defects in immature NPCs, rather than post-mitotic neurons, consistently observing that at the level of gene pathways and networks, gene expression differences identified in patient neurons are frequently conserved in patient NPCs $(13,24)$.

NPCs are a scalable cell type, amenable to parallel culture of dozens of cell lines and highly adaptable to automated methods. They are straightforward to maintain in vitro, requiring less frequent feeding and passaging than their source hiPSCs (25). NPCs proliferate robustly, are cryopreservable, and easily differentiated or induced to mature neurons $(25,26)$ and astrocytes $(27)$. Overall, NPCs 
are an ideal cell type for mechanistic studies of disease biology as well as adaptation to high throughput drug screens.

Current hiPSC models of neuropsychiatric disease have generally focused on those risk factors encoded in the nuclear DNA sequence, with little consideration of epigenetic regulation and no understanding of mitochondrial biology [reviewed in (28)]. While it is well established that highly penetrant nuclear genome mutations can recapitulate neuropsychiatric disease biology, it has been unclear, to date, to what extent hiPSC-based models also capture the effects of mitochondrial disease risk. In fact, although it was previously established that mitochondria undergo morphological and metabolic reconfigurations while donor cells are being reprogrammed to hiPSCs (29) and hypothesized that mitochondrial state was linked to cellular differentiation (30), it was unclear to what extent cell-type specific mitochondria activity patterns would be re-established during neuronal differentiation.

Now, Lorenz et al demonstrate that disease- and genotype-specific mitochondrial risk effects can also be modeled with hiPSCs. Over the course of neuronal differentiation from hiPSCs, mitochondria shift toward a neuronal-like oxidative metabolism. hiPSC-derived NPCs derived from three patients with homoplasmic mitochondrial mutations in MT-ATP6 not only retained the mutant genotype, but also exhibited disease relevant phenotypes such as decreased ATP production, abnormally high mitochondrial membrane potential, and altered calcium homeostasis (31). Moreover, as a proof-of-concept, they successfully screened 130 drugs on NPCs derived from one of these patients, identifying ten compounds that significantly reduced mitochondrial membrane potential (31). While it is premature to speculate whether these drugs represent novel therapeutics for mitochondrial disease, this is an exciting demonstration that hiPSC-based models can provide novel insights into mitochondrial disorders.

A critical limitation of hiPSC-based models is that the genotype in the donor somatic cells (typically skin or blood) accurately reflects what is observed in the tissue impacted by disease. At the level of nuclear DNA, hiPSCbased models fail to accurately model the impact of somatic mosaicism, either because: (I) the genotype of the donor cells is different from the brain; (II) the hiPSC-derived neural cells spontaneously differ from that of the donor cells (32); or (III) the mosaic variants are selected for or against during the reprogramming or differentiation processes causing the hiPSC-derived neural composition to inaccurately reflect that found in the brain (33). Variation between mitochondrial populations in donor cells, disease cells and hiPSC-derived populations remains a similar concern for mitochondrial disorders. Although it is clear that a homoplasmic mitochondrial population can be accurately maintained and modeled using hiPSC-based models (31), the extent to which that is true for heteroplasmic mitochondrial disorders is unknown.

Since the first discovery that patient somatic cells can be reprogrammed to hiPSCs that are theoretically capable of generating all the cell types of the human body, our ability to model the impact of genetic risk on disease phenotypes continues to advance. Now, with the knowledge that mutations in either the nuclear or the mitochondrial genome can be modeled as well as screened against using hiPSCs, our ability to uncover novel disease mechanisms and therapeutics for neuropsychiatric disease continues to expand.

\section{Acknowledgements}

Funding: KJ Brennand is a New York Stem Cell FoundationRobertson Investigator. This work was partially supported by National Institute of Health grants R01 MH101454, as well as the New York Stem Cell Foundation and the Brain Behavior Research Foundation.

\section{Footnote}

Conflicts of Interest: The author has no conflicts of interest to declare.

\section{References}

1. Takahashi K, Tanabe K, Ohnuki M, et al. Induction of pluripotent stem cells from adult human fibroblasts by defined factors. Cell 2007;131:861-72.

2. Mariani J, Coppola G, Zhang P, et al. FOXG1-dependent dysregulation of GABA/glutamate neuron differentiation in autism spectrum disorders. Cell 2015;162:375-90.

3. Brennand KJ, Simone A, Jou J, et al. Modelling schizophrenia using human induced pluripotent stem cells. Nature 2011;473:221-5.

4. Kondo T, Asai M, Tsukita K, et al. Modeling Alzheimer's disease with iPSCs reveals stress phenotypes associated with intracellular Abeta and differential drug responsiveness. Cell Stem Cell 2013;12:487-96.

5. Nguyen HN, Byers B, Cord B, et al. LRRK2 Mutant 
iPSC-Derived DA neurons demonstrate increased susceptibility to oxidative stress. Cell Stem Cell 2011;8:267-80.

6. Tang H, Hammack C, Ogden SC, et al. Zika virus infects human cortical neural progenitors and attenuates their growth. Cell Stem Cell 2016;18:587-90.

7. McLaren D, Gorba T, Marguerie de Rotrou A, et al. Automated large-scale culture and medium-throughput chemical screen for modulators of proliferation and viability of human induced pluripotent stem cellderived neuroepithelial-like stem cells. J Biomol Screen 2013;18:258-68.

8. Zhao WN, Cheng C, Theriault KM, et al. A highthroughput screen for Wnt/beta-catenin signaling pathway modulators in human iPSC-derived neural progenitors. J Biomol Screen 2012;17:1252-63.

9. Xu M, Lee EM, Wen Z, et al. Identification of smallmolecule inhibitors of Zika virus infection and induced neural cell death via a drug repurposing screen. Nat Med 2016;22:1101-7.

10. Pei Y, Peng J, Behl M, et al. Comparative neurotoxicity screening in human iPSC-derived neural stem cells, neurons and astrocytes. Brain Res 2016;1638:57-73.

11. Efthymiou A, Shaltouki A, Steiner JP, et al. Functional screening assays with neurons generated from pluripotent stem cell-derived neural stem cells. J Biomol Screen 2014;19:32-43.

12. Yang YM, Gupta SK, Kim KJ, et al. A small molecule screen in stem-cell-derived motor neurons identifies a kinase inhibitor as a candidate therapeutic for ALS. Cell Stem Cell 2013;12:713-26.

13. Brennand K, Savas JN, Kim Y, et al. Phenotypic differences in hiPSC NPCs derived from patients with schizophrenia. Mol Psychiatry 2015;20:361-8.

14. Mariani J, Simonini MV, Palejev D, et al. Modeling human cortical development in vitro using induced pluripotent stem cells. Proc Natl Acad Sci U S A 2012;109:12770-5.

15. Nicholas CR, Chen J, Tang Y, et al. Functional maturation of hPSC-derived forebrain interneurons requires an extended timeline and mimics human neural development. Cell Stem Cell 2013;12:573-86.

16. Pasca AM, Sloan SA, Clarke LE, et al. Functional cortical neurons and astrocytes from human pluripotent stem cells in 3D culture. Nat Methods 2015;12:671-8.

17. Sareen D, Ebert AD, Heins BM, et al. Inhibition of apoptosis blocks human motor neuron cell death in a stem cell model of spinal muscular atrophy. PLoS One 2012;7:e39113.
18. Marchetto MC, Belinson H, Tian Y, et al. Altered proliferation and networks in neural cells derived from idiopathic autistic individuals. Mol Psychiatry 2017;22:820-35.

19. Topol A, Zhu S, Hartley BJ, et al. Dysregulation of miRNA-9 in a Subset of schizophrenia patient-derived neural progenitor cells. Cell Rep 2016;15:1024-36.

20. Srikanth P, Han K, Callahan DG, et al. Genomic DISC1 Disruption in hiPSCs Alters Wnt signaling and neural cell fate. Cell Rep 2015;12:1414-29.

21. Madison JM, Zhou F, Nigam A, et al. Characterization of bipolar disorder patient-specific induced pluripotent stem cells from a family reveals neurodevelopmental and mRNA expression abnormalities. Mol Psychiatry 2015;20:703-17.

22. Liu GH, Qu J, Suzuki K, et al. Progressive degeneration of human neural stem cells caused by pathogenic LRRK2. Nature 2012;491:603-7.

23. An MC, Zhang N, Scott G, et al. Genetic correction of huntington's disease phenotypes in induced pluripotent stem cells. Cell Stem Cell 2012;11:253-63.

24. Hoffman GE, Hartley BJ, Flaherty E, et al. Transcriptional signatures of schizophrenia in hiPSC-derived NPCs and neurons are concordant with signatures from post mortem adult brains. bioRxiv 2017. [Epub ahead of print].

25. Topol A, Tran NN, Brennand KJ. A guide to generating and using hiPSC derived NPCs for the study of neurological diseases. J Vis Exp 2015:e52495.

26. Ho SM, Hartley BJ, Tcw J, et al. Rapid Ngn2-induction of excitatory neurons from hiPSC-derived neural progenitor cells. Methods 2016;101:113-24.

27. Tcw J, Wang M, Pimenova AA, et al. An efficient platform for astrocyte differentiation from human induced pluripotent stem cells. Stem Cell Reports 2017;9:600-14.

28. Soliman MA, Aboharb F, Zeltner N, et al. Pluripotent stem cells in neuropsychiatric disorders. Mol Psychiatry 2017;22:1241-9.

29. Prigione A, Fauler B, Lurz R, et al. The senescencerelated mitochondrial/oxidative stress pathway is repressed in human induced pluripotent stem cells. Stem Cells 2010;28:721-33.

30. Prigione A, Adjaye J. Modulation of mitochondrial biogenesis and bioenergetic metabolism upon in vitro and in vivo differentiation of human ES and iPS cells. Int J Dev Biol 2010;54:1729-41.

31. Lorenz C, Lesimple P, Bukowiecki R, et al. Human iPSCderived neural progenitors are an effective drug discovery model for neurological mtDNA Disorders. Cell Stem Cell 2017;20:659-74.e9. 
32. McConnell MJ, Lindberg MR, Brennand KJ, et al. Mosaic copy number variation in human neurons. Science 2013;342:632-7.

doi: $10.21037 /$ sci.2017.10.05

Cite this article as: Brennand KJ. Personalized medicine in a dish: the growing possibility of neuropsychiatric disease drug discovery tailored to patient genetic variants using stem cells. Stem Cell Investig 2017;4:91.
33. Tcw J, Carvalho CM, Yuan B, et al. Divergent levels of marker chromosomes in an hipsc-based model of psychosis. Stem Cell Reports 2017;8:519-28. 